\title{
Strangeness Production in Deep-Inelastic ep Scattering at HERA
}

\section{Khurelbaatar BEGZSUREN* ${ }^{* *}$}

Institute of Physics and Technology of the Mongolian Academy of Sciences, Ulaanbaatar,

Mongolia

E-mail: hurel@mail.desy.de

\section{On behalf of the $\mathrm{H} 1$ collaboration}

The production of neutral strange hadrons is studied using deep-inelastic events measured with the H1 detector at HERA. The measurements of $K_{s}^{0}$ and $\Lambda(\bar{\Lambda})$ productions are made in two regions of phase space defined by the negative four-momentum transferred squared of the photon, $7<$ $Q^{2}<100 \mathrm{GeV}^{2}$ and the inelasticity $0.1<y<0.6$ for the $K_{s}^{0}$ and $145<Q^{2}<20000 \mathrm{GeV}^{2}$ and $0.2<y<0.6$ for the $\Lambda . K_{s}^{0}$ and $\Lambda(\bar{\Lambda})$ production cross sections are determined. Differential ratios of $K_{s}^{0}$ production to charged hadron production are measured. Differential $\Lambda(\bar{\Lambda})$ yields per event are determined. The $\Lambda-\bar{\Lambda}$ asymmetry is measured and found to be consistent with zero. Predictions of leading order Monte Carlo programs are compared to data.

XXI International Workshop on Deep-Inelastic Scattering and Related Subjects -DIS2013, 22-26 April 2013

Marseille, France

* Speaker.

$\dagger$ address: DESY, Notkestarße 85, D-22603 Hamburg, Germany 


\section{Introduction}

The measurement of strange particle production in high energy collisions provides valuable information for understanding Quantum Chromodynamics (QCD) in the perturbative and nonperturbative regime. In neutral current deep-inelastic ep scattering (DIS) at HERA four different processes depicted in figure 1 contribute to strange hadron production. Strange quarks may origi-

(a)

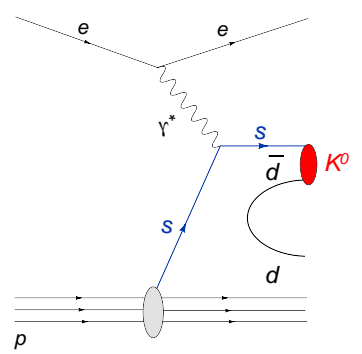

(b)

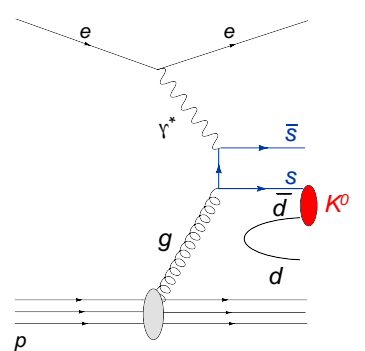

(c)

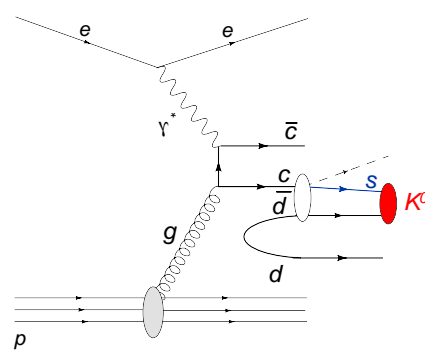

(d)

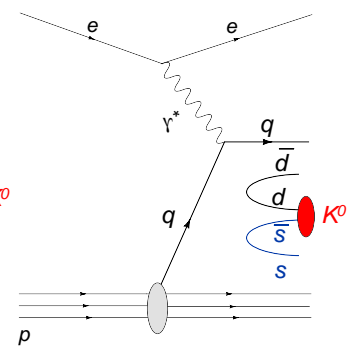

Figure 1: Schematic diagrams for the processes contributing to strangeness production in ep scattering: (a) direct production from the strange sea, (b) BGF, (c) heavy hadron decays and (d) fragmentation. The diagrams relevant for $K^{0}$ production are shown.

nate directly from the strange sea of the proton (figure 1a), from boson-gluon-fusion (BGF, figure $1 b$ ), from the decays of heavy flavoured hadrons (figure 1c) and from the creation of $s \bar{s}$ pairs in the non-perturbative fragmentation process (figure 1d). The latter process is the dominant source for strange hadron production. In the modelling of the fragmentation process the suppression of $s \bar{s}$ pairs due to the mass of the strange quark is generally controlled by the strangeness suppression factor $\lambda_{s}[1,2]$. Especially, the ratio of $K_{s}^{0}$ to charged particles should strongly depends on this quark mass effect.

This paper presents new measurements of $K_{s}^{0}$ production at low $Q^{2}$ and $\Lambda$ production at high $Q^{2}$. Results are presented on $K_{s}^{0}$ and $\Lambda$ production cross sections, on the ratio of $K_{s}^{0}$ production to charged particles production measured in the same phase space region, on $\Lambda$ yields normalised to DIS cross sections, and on the $\Lambda-\bar{\Lambda}$ asymmetry. The measurements are shown as a function of several observables characterising the DIS kinematics and the strange particles production dynamics in the laboratory frame. The results are compared with predictions obtained from leading order Monte Carlo calculations, based on matrix elements with parton shower simulation. The rôle of strangeness suppression on hadrons with strangeness is investigated.

\section{Selection of Hadron Candidates}

The data used in the analyses correspond to an integrated luminosity of $109 \mathrm{pb}^{-1}$ in case of $K_{s}^{0}$ production and $340 \mathrm{pb}^{-1}$ in case of $\Lambda$ production and were collected with the $\mathrm{H} 1$ detector [3] in the years 2004 to 2007 when protons with an energy of $920 \mathrm{GeV}$ collided with electrons ${ }^{1}$ with an energy of $27.6 \mathrm{GeV}$ producing a centre-of-mass energy of $\sqrt{s}=319 \mathrm{GeV}$. The kinematics of the scattering process at HERA are described using the Lorentz invariant variables $Q^{2}$ denoting

\footnotetext{
${ }^{1}$ in this paper "electron" is used to denote both electron and positron
} 
the square of the photon virtuality, the inelasticity in the proton rest frame $y$ and Bjorken scaling variable $x$. At fixed $\sqrt{s}$ only two of these variables are independent because of $Q^{2}=x y s$. The following kinematic ranges are used in the analyses: $7<Q^{2}<100 \mathrm{GeV}^{2}$ and $0.1<y<0.6$ for the $K_{s}^{0}$ and $145<Q^{2}<20000 \mathrm{GeV}^{2}$ and $0.2<y<0.6$ for the $\Lambda$ (see Table 1 ).

\begin{tabular}{|c|c|c|}
\hline & DIS kinematics & Hadron kinematics \\
\hline$K_{s}^{0}$ & $7<Q^{2}<100 \mathrm{GeV}^{2}, 0.1<y<0.6$ & $0.5<p_{T}<3.5 \mathrm{GeV},-1.3<\eta<1.3$ \\
\hline$\Lambda$ & $145<Q^{2}<20000 \mathrm{GeV}^{2}, 0.2<y<0.6$ & $p_{T}>0.3 \mathrm{GeV},-1.5<\eta<1.5$ \\
\hline
\end{tabular}

Table 1: Phase space regions explored in the analyses of $K_{s}^{0}$ and $\Lambda$ production, respectively.

The $K_{s}^{0}$ mesons and $\Lambda$ baryons ${ }^{2}$ are measured by the kinematic reconstruction of their decays $K_{s}^{0} \rightarrow \pi^{+} \pi^{-}$and $\Lambda \rightarrow p \pi^{-}$, respectively. The number of $K_{s}^{0}$ mesons and $\Lambda$ baryons is obtained by fitting the invariant mass spectra with the sum of a signal and background function. For the signal function the skewed t-student function is used while the background shape is described by a threshold function with exponential damping. In total approximately $290000 K_{s}^{0}$ mesons and 7000 $\Lambda(\bar{\Lambda})$ baryons are reconstructed in the phase space given in table 1 . The fitted $K_{s}^{0}$ and $\Lambda$ masses agree with the world average [4].

\section{Results and Discussion}

\subsection{Inclusive Cross Sections}

The visible inclusive production cross sections $\sigma_{v i s}$ measured in the kinematic region defined in Table 1, are

$$
\begin{aligned}
\sigma_{v i s}\left(e p \rightarrow e K_{s}^{0} X\right) & =10.66 \pm 0.02 \text { (stat. })_{-8.5}^{+9.4} \text { (syst.) nb } \\
\sigma_{v i s}(e p \rightarrow e \Lambda X) & =144.7 \pm 0.04(\text { stat. })_{-8.5}^{+9.4} \text { (syst.) } \mathrm{pb}
\end{aligned}
$$

Using a strangeness suppression factor of $\lambda_{s}=0.286$ the models RAPGAP [5] and DJANGOH [6] predict $K_{s}^{0}$ cross sections of $10.93 \mathrm{nb}$ and $9.88 \mathrm{nb}$, respectively, in reasonable agreement with the measurement. The cross section predictions for $\Lambda+\bar{\Lambda}$ production from the MEPS and CDM [7]models are shown in Table 2 for two values of the strangeness suppression parameter $\lambda_{s}$. The measured inclusive $\Lambda+\bar{\Lambda}$ cross section is close to the CDM prediction with $\lambda_{s}=0.22$ and to the MEPS prediction with $\lambda_{s}=0.286$.

\subsection{Differential cross sections}

Differential cross sections of $K_{s}^{0}$ and $\Lambda$ production are shown in figures 2a,b and $3 \mathrm{a}$ as a function of $Q^{2}$, and as a function of the kinematic variable of the neutral strange hadrons in the laboratory frame, $\eta$ along with the predictions of the MEPS and CDM models. The cross sections fall

\footnotetext{
${ }^{2}$ Unless otherwise noted, charge conjugate states are always implied.
} 


\begin{tabular}{|c|c|c|}
\hline & $\lambda_{s}=0.220$ & $\lambda_{s}=0.286$ \\
\hline$\sigma_{v i s}(e p \rightarrow e[\Lambda+\bar{\Lambda}] X) \mathrm{CDM}$ & $136 \mathrm{pb}$ & $161 \mathrm{pb}$ \\
\hline$\sigma_{\text {vis }}(e p \rightarrow e[\Lambda+\bar{\Lambda}] X) \mathrm{MEPS}$ & $120 \mathrm{pb}$ & $144 \mathrm{pb}$ \\
\hline
\end{tabular}

Table 2: Monte Carlo predictions for different settings of the strangeness suppresion factor $\lambda_{s}$.

rapidly as $Q^{2}$ grows. The figures also include the ratios of predicted to measured cross sections for a better shape comparison. Apart from small normalisation differences the models describe the shapes of the measured cross sections as a function of $Q^{2}$ and $\eta$ reasonably well.
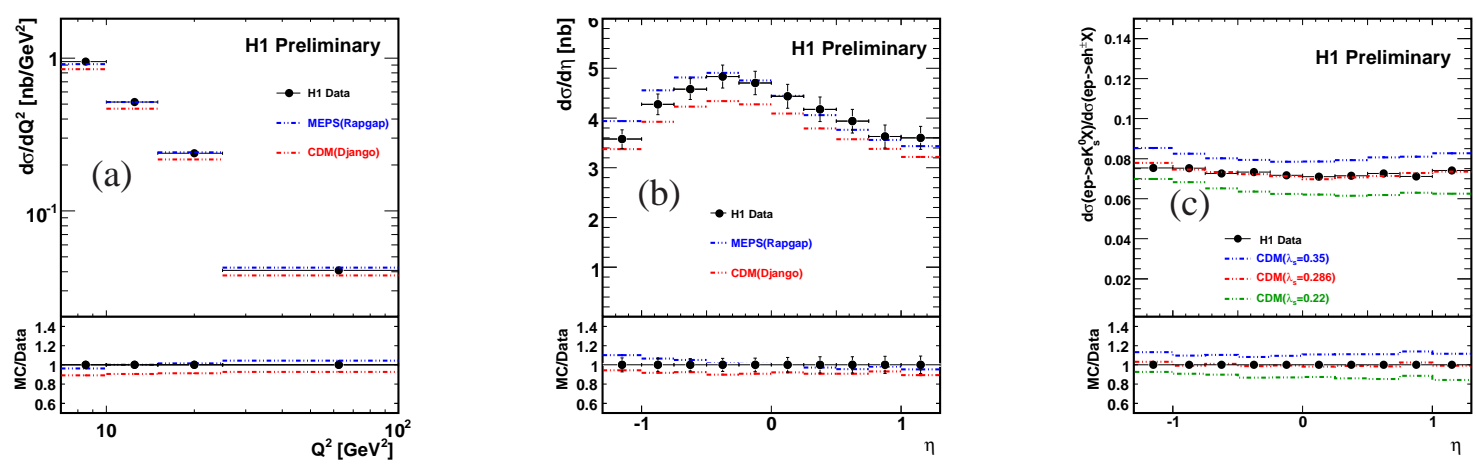

Figure 2: Differential $K_{s}^{0}$ production cross sections as a function of (a) the photon virtuality squared $Q^{2}$, (b) its pseudorapidity $\eta$ and (c) ratio of $K_{s}^{0}$ to charged particle production as a function of $\eta$ in comparison to RAPGAP (MEPS) and DJANGOH (CDM). The inner (outer) error bars show the statistical (total) errors. The ratios "MC/Data" are shown for the different Monte Carlo predictions. For comparison, the data points are put to one.

\subsection{Ratio of $K_{s}^{0}$ Production to Charged Particle Production}

By normalising the $K_{s}^{0}$ production cross section to the cross section of charged particle production many model dependent uncertainties, like the cross section dependence on proton PDFs, cancel thus enhancing the sensitivity to details of the fragmentation process. In Figure $2 \mathrm{c}$ the ratio of $K_{s}^{0}$ production to the cross section charged particle procduction is shown as a function of $\eta$ in comparison to the expectations from DJANGOH using three different values of $\lambda_{s}$ ranging from 0.220 to 0.35 . The ratio in $\eta$ is well described by the model in shape and a high sensitivity on $\lambda_{s}$ is observed in the absolute value of this ratio, demonstrating the clear potential of using this ratio for extracting the strangeness suppression factor $\lambda_{s}$.

\section{4 $\Lambda$ Production to DIS Cross Section Ratio}

In Figure $3 \mathrm{~b}$ the ratio of $\Lambda$ production to DIS cross section is shown as a function of $Q^{2}$ in 
comparison to the expectations from RAPGAP and DJANGOH both using $\lambda_{s}=0.286$ and $\lambda_{s}=$ 0.220. The DJANGOH prediction with $\lambda_{s}=0.286$ yields the worst description of the data by overshooting them significantly independent of $Q^{2}$. For the same strangeness suppression factor also RAPGAP tends to yield ratios larger than observed in data for $Q^{2}<200 \mathrm{GeV}^{2}$. The best description is provided by DJANGHO using $\lambda_{s}=0.220$.
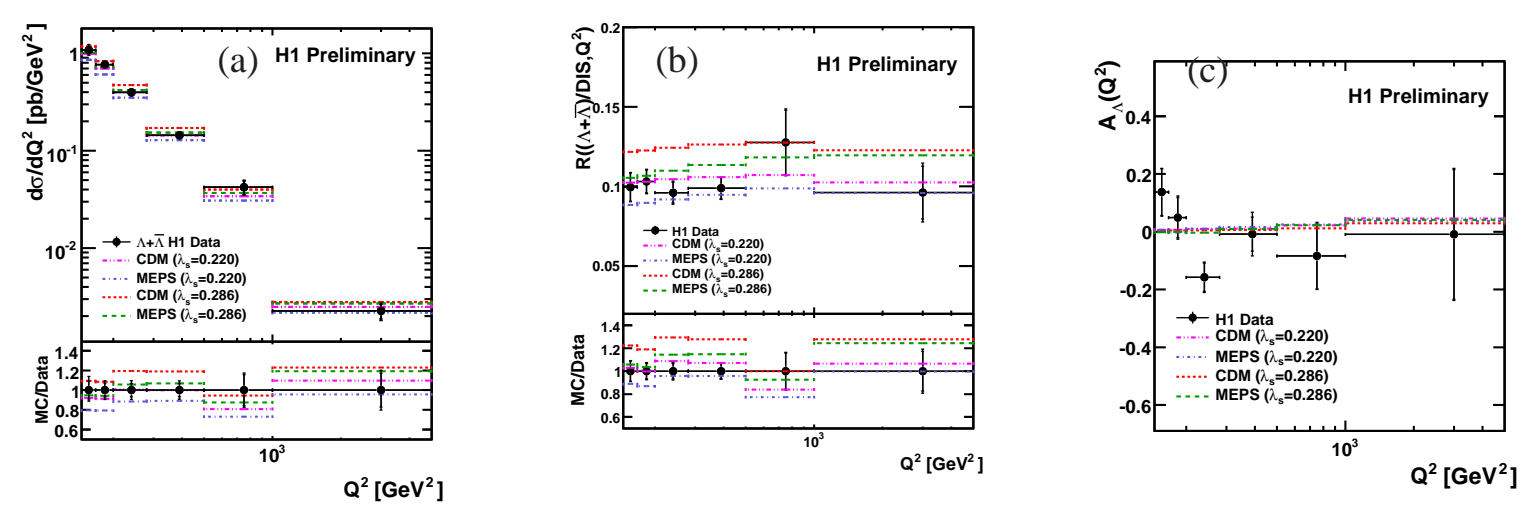

Figure 3: The $Q^{2}$ dependence of (a) differential $\Lambda$ production cross section, (b) ratio $\mathrm{R}(\mathrm{DIS})$ of $\Lambda$ production to DIS cross section and (c) asymmetry $A_{\Lambda}$ in comparison to RAPGAP (MEPS) and DJANGOH (CDM) with two different values of $\lambda_{s}$. The inner (outer) error bars show the statistical (total) errors. The "MC/Data" ratios are shown for different Monte Carlo predictions. For the ratios the data points are put at one for comparison.

\section{$3.5 \Lambda-\bar{\Lambda}$ Asymmetries}

The $\Lambda-\bar{\Lambda}$ asymmetry is defined as:

$$
A_{\Lambda}=\frac{\sigma_{v i s}(e p \rightarrow e \Lambda X)-\sigma_{v i s}(e p \rightarrow e \bar{\Lambda} X)}{\sigma_{v i s}(e p \rightarrow e \Lambda X)+\sigma_{v i s}(e p \rightarrow e \bar{\Lambda} X)}
$$

This observable could shed light on the mechanism of baryon number transfer in $e p$ scattering. A significant positive asymmetry would be an indication for the baryon number transfer from the proton to the $\Lambda$ baryon. If present such an effect should be more pronounced in the positive $\eta$ region in the laboratory frame. For the kinemaic region defined in table 1 the asymmetry is measured to be $\mathrm{A}_{\Lambda}=0.002 \pm 0.022$ (stat.) \pm 0.018 (syst.). In figure $3 \mathrm{c}$ the asymmetry $A_{\Lambda}$ is shown as a function of $Q^{2}$. The data show no evidence for a non-vanishing asymmetry in the phase space region investigated.

\section{Conclusions}

This paper presents a study of inclusive production of $K_{s}^{0}$ and $\Lambda$ in DIS at low $Q^{2}$ and high $Q^{2}$ measured with the H1 detector at HERA. The cross sections of $K_{s}^{0}$ and $\Lambda$ production are measured as a function of the DIS kinematic variable $Q^{2}$ and of strange hadron production variables in the laboratory frame. In addition results on the ratio of $K_{s}^{0}$ production cross section to the charged 
particle cross section, the $\Lambda$ production to DIS cross section ratio and the $\Lambda-\bar{\Lambda}$ asymmetry are presented. The measurements are compared to model predictions of DJANGOH, based on the colour-dipol model (CDM) and RAPGAP based on DGLAP matrix element calculations supplemented with parton showers (MEPS). Within the uncertainties both models provide a reasonable description of the data. The sensitivity of the ratio of $K_{s}^{0}$ to charged particle production cross sections on the strangeness suppression factor $\lambda_{s}$ is demonstrated, however, a detailed understanding of concurrent processes of $K_{s}^{0}$ production is mandatory prior to the determination of $\lambda_{s}$. The measured visible $\Lambda$ cross section is found to be described best by the CDM using $\lambda_{s}=0.220$ and the MEPS model using $\lambda_{s}=0.286$. When investigating the $\Lambda$ production to DIS cross section ratio the best agreement is observed for the CDM with $\lambda_{s}=0.220$. The $\Lambda-\bar{\Lambda}$ asymmetry is found to be consistent with zero.

\section{References}

[1] T. Sjöstrand, "High-energy physics event generation with PYTHIA 5.7 and JETSET 7.4," Comput. Phys. Commun. 82 (1994) 74, JETSET version 7.4 is used.

[2] T. Sjöstrand, "The Lund Monte Carlo For Jet Fragmentation And E+ E- Physics: Jetset Version 6.2," Comput. Phys. Commun. 39 (1986) 347;

T. Sjöstrand and M. Bengtsson, "The Lund Monte Carlo For Jet Fragmentation And E+ E- Physics. Jetset Version 6.3: An Update," Comput. Phys. Commun. 43 (1987) 367;

B. Andersson et al. "Parton Fragmentation And String Dynamics," Phys. Rept. 97 (1983) 31.

[3] I. Abt et al. [H1 Collaboration], "The H1 detector at HERA," Nucl. Instrum. Meth. A 386 (1997) 310; I. Abt et al. [H1 Collaboration], "The Tracking, calorimeter and muon detectors of the H1 experiment at HERA,” Nucl. Instrum. Meth. A 386 (1997) 348.

[4] K. Nakamura et al. (Particle Data Group), J. Phys. G 37, 075021 (2010).

[5] H. Jung, "Hard diffractive scattering in high-energy e p collisions and the Monte Carlo generator RAPGAP," Comp. Phys. Commun. 86 (1995) 147.

[6] G. A. Schuler and H. Siesberger, DJANGO, Proceedings of "Physics at HERA", eds. W. Buchmüller and G. Ingelman, DESY, Hamburg (1992) 1419.

[7] B. Andersson et al. "Coherence Effects in Deep Inelastic Scattering," Z. Phys. C 43 (1989) 625; L. Lönnblad, "Rapidity gaps and other final state properties in the colour dipole model for deep inelastic scattering," Z. Phys. C 65 (1995) 285. 